\title{
Um Algoritmo Evolutivo Baseado em Chaves Aleatórias Viciadas Aplicado ao Problema de Roteamento de Veículos com Janelas de Tempo
}

\author{
Welton T. M. de Sousa* Carlos A. Silva \\ Instituto Federal de Minas Gerais - Departamento de Computação, \\ 34515-640, Sabará, MG \\ E-mail: weltonthiago7@hotmail.com, carlos.silva@ifmg.edu.br.
}

\section{RESUMO}

O Problema de Roteamento de Veículos (PRV), introduzido na literatura por [1], é um dos mais clássicos problemas da pesquisa operacional. A forma mais simples do PRV é o Problema de Roteamento de Veículos Capacitados (PRVC), onde uma frota de veículos, localizada inicialmente em um depósito, deve atender a um conjunto de consumidores com diferentes demandas de produtos a serem distribuídos por essa frota. Neste trabalho abordaremos o Problema de Roteamento de Veículos com Janelas de Tempo (PRVJT), o qual é uma extensão do PRVC. Além da restrição de capacidade, são adicionadas restrições relacionadas ao horário em que cada consumidor exige ser atendido. Para cada consumidor $i$, é associado um intervalo de tempo ou janela de tempo $\left[a_{i}, b_{i}\right]$ indicando o horário de início do atendimento, e um tempo de serviço $s_{i}$, determinando o período de tempo que o veículo deve aguardar a finalização das tarefas. O algoritmo evolutivo proposto para resolver este problema, consiste em determinar um conjunto de rotas com o menor custo possível, respeitando um determinado conjunto de restrições.

Neste trabalho propomos um método de solução para o PRVJT utilizando um algoritmo genético baseado em chaves aleatórias viciadas, denominado BRKGA (Biased Random-key Genetic Algorithm). Representamos a solução do problema por um vetor de $n$ chaves aleatórias, sendo essas chaves números reais dentro do intervalo $[0,1)$. Um decodificador é usado para mapear o vetor de chaves aleatórias e transformá-lo numa solução do PRVJT, ou seja, em um vetor de números inteiros, em que subsequências desses números correspondem a rotas formadas pelas cidades da instância utilizada. Inicialmente é gerada uma população de $p$ vetores de chaves aleatórias e em seguida são selecionadas as melhores soluções. O conjunto de tamanho $p_{e}$ contendo as melhores soluções é preservado para a próxima geração ou iteração do algoritmo. Um novo conjunto de tamanho $p-p_{e}$ é adicionado às melhores soluções, compondo a nova população da próxima geração. Este novo conjunto de soluções é gerado a partir de combinações entre pais e filhos conforme uma dada distribuição probabilística.

De acordo com [2], um RKGA (Random Key Genetic Algorithm) evolui uma população, de $p$ vetores de chaves aleatórias aplicando o princípio de Darwin, ou seja, há uma maior probabilidade de que os indivíduos mais aptos sobrevivam. Uma população inicial de $p$ vetores de $n$ chaves aleatórias é gerada de forma randômica. $\mathrm{Na} k$-ésima geração, a população é particionada em dois conjuntos: $p_{e}<p / 2 \mathrm{e}$ $p_{n e}=p-p_{e}$, conjunto elite e não-elite, respectivamente. O conjunto $p_{e}$ é constituído dos vetores que formam as melhores soluções, e consequentemente o conjunto $p_{n e}$ é formado pelo restante da população. Para a $k+1$-ésima geração, a nova população é formada pelos conjuntos $p_{e}, p_{m}$ e $p_{r}$. O conjunto $p_{m}$ é composto por vetores de chaves aleatórias, e, é denominado de mutante, pois desempenha o mesmo papel dos operadores de mutação nos algoritmos genéticos clássicos, ou seja, evita que a população estacione em um ótimo local. O conjunto $p_{r}=p-p_{e}-p_{m}$ complementa a população. Os vetores deste último conjunto são gerados combinando pares de soluções da população da $k$-ésima geração,

${ }^{*}$ Bolsista de Iniciação Científica PIBIC/IFMG 
ambos escolhidos aleatoriamente, com a combinação uniforme seguindo os parâmetros de [4]. Sejam $s=\left[0 . . s_{i} . .0\right]$ e $t=\left[0 . . t_{i} . .0\right]$ dois vetores-pai e $u=\left[0 . . u_{i} . .0\right]$ um vetor-filho, onde 0 representa o depósito e $s_{i}, t_{i}, u_{i}$ representam chaves aleatórias que indicam alguma cidade no PRVJT. O elemento $u_{i}$ pode receber $s_{i}$ ou $t_{i}$ de acordo com as probabilidades $\rho_{s}$ e $\rho_{t}=1-\rho_{s}$, respectivamente.

$\mathrm{O}$ algoritmo BRKGA-PRVJT proposto, está sendo implementado em linguagem $\mathrm{C}++$, utilizando o compilador NetBeans IDE 7.31, e executado em um computador Intel(R) Core i3 $2.5 \mathrm{GHz}$, com $3 \mathrm{~GB}$ de memória RAM, sob plataforma Windows 7 Professional 32 bits. Estão sendo utilizadas, para teste, as instâncias introduzidas por [3], usadas amplamente como referência de desempenho de algoritmos para o PRVJT. Para a implementação do BRKGA-PRVJT foi utilizado o modelo matemático apresentado em [5]. A função de avaliação foi definida como:

$$
f(s)=\mu n_{v}(s)+\alpha e_{c}(s)+\beta v_{r}(s)+\sum_{(r, s) \in \mathcal{A}} d_{r s},
$$

onde, para cada solução $s$, o número de veículos é representado por $n_{v}$; a soma de excessos de capacidade de todos os veículos é indicada por $e_{c}$ e a soma das violações referentes às restrições de janela de tempo é representada por $v_{r}$. Além disso, a variável $d_{r s}$ representa a distância entre os consumidores $r$ e $s$, e $\mathcal{A}$ é o conjunto dos arcos da solução. O Algoritmo 1 apresenta o pseudocódigo do BRKGA aplicado ao PRVJT.

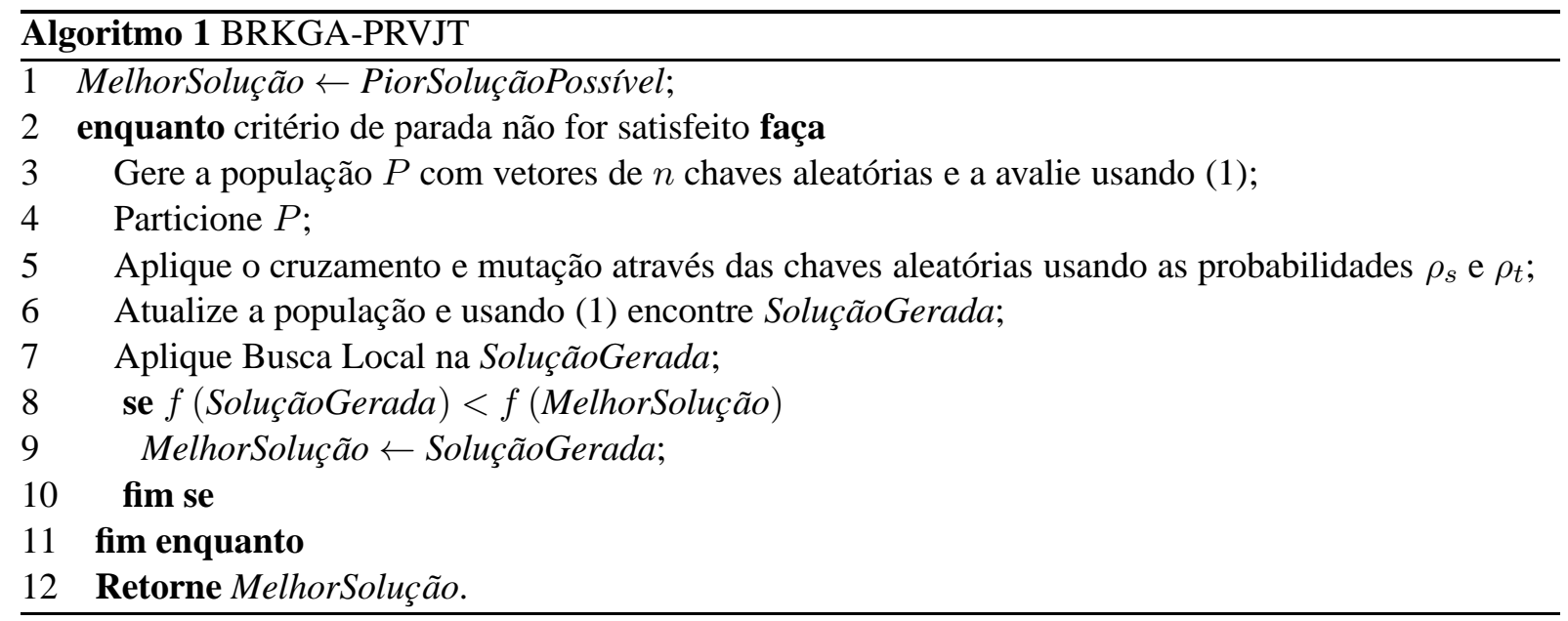

Esta pesquisa se encontra em fase de simulação. A metodologia de chaves aleatórias pode proporcionar uma nova forma de explorar o espaço de soluções para o PRVJT, além de que, as características probabilísticas facilitariam a não estagnação das soluções em ótimos locais.

Palavras-chave: Problemas de Roteamento de Veículos, Algoritmo Evolutivo, Chaves Aleatórias.

\section{Referências}

[1] G.B. Dantzig e J.H. Ramser, The truck dispatching problem, Management Science, (1959) 80-91.

[2] M.G.C. Resende, Introdução aos algoritmos genéticos de chaves aleatórias viciadas, em "Simpósio Brasileiro de Pesquisa Operacional”, pp. 16-19, 2013.

[3] M.M. Solomon, Algorithms for vehicle routing and scheduling problems with time windows constraints, European Journal of Operational Research, 35 (1987) 254-266.

[4] W.M. Spears e K.A. DeJong, On the virtues of parameterized uniform crossover, em "Proceedings of the Fourth International Conference on Genetic Algorithms", pp. 230-236, 1991.

[5] K.C. Tan; L.H. Lee; Q.L. Zhu e K. Ou, Heuristic methods for vehicle routing problem with time windows, Artificial Inteligence in Engineering, 15 (2001) 281-295. 